\title{
Comparación del Desempeño de Estimadores de Estado no Lineales para Determinar la Concentración de Biomasa y Sustrato en un Bioproceso
}

\author{
Fabián A. Ortega ${ }^{(1)}$, Omar A. Pérez ${ }^{(2)}$ y Emiro A. López ${ }^{(2)}$ \\ (1) Universidad Nacional de Colombia. Escuela de Procesos y Energía. Facultad de Minas. Grupo de \\ investigación en Procesos Dinámicos KALMAN. Cra. 80 x Cl. 65. Barrio Robledo. Medellín - Antioquia, \\ Colombia. (e-mail: faortegaqui@unal.edu.co). \\ (2) Universidad de Córdoba. Facultad de Ingeniería. Programa de Ingeniería de Alimentos. Grupo de \\ investigación GIPPAL. Carrera 6 No. 76-103. Montería - Córdoba, Colombia \\ (e-mail: operez@correo.unicordoba.edu.co,ealopez@correo.unicordoba.edu.co).
}

Recibido Abr. 27, 2015; Aceptado Jun. 2, 2015; Versión final Jul. 15, 2015, Publicado Oct. 2015

\begin{abstract}
Resumen
Este trabajo presenta un análisis comparativo de varias técnicas de estimación no lineal cuando es aplicada a un bioproceso. El observador Luenberger extendido, el filtro de Kalman extendido y el observador de modos deslizantes fueron evaluados para un proceso de crecimiento microbiano. El desempeño de los estimadores no lineales es evaluado en términos de indicadores de error y su habilidad para hacer frente con incertidumbres del proceso tales como errores de medición e incertidumbre de las condiciones iniciales. Los resultados de la simulación sugieren que el filtro de Kalman extendido provee estimaciones precisas de los estados y que el Observador de Luenberger Extendido no es recomendable para este tipo de bioprocesos.
\end{abstract}

Palabras clave: filtro; observador; modos deslizantes; estimadores de estado; bioproceso

\section{Performance Comparison of Nonlinear Estimators of State for Determining Biomass Concentration and Substrate in a Bioprocess}

\begin{abstract}
This paper presents a comparative analysis of various nonlinear estimation techniques when is applied on a bioprocess. Extended Luenberger observer, Extended Kalman filter and Sliding Mode observer were evaluated for a microbial growth process. The performance of the nonlinear estimators is evaluated in terms of error indicators and their ability to cope with process uncertainties such as measurement errors and uncertain initial conditions. The simulation results suggest that the extended Kalman filter provide accurate estimates of the states and that the Extended Luenberger observer is not recommended for this type of bioprocesses.
\end{abstract}

Keywords: filter; observer; sliding mode; estimators of state; bioprocess 


\section{INTRODUCCIÓN}

Los modelos de bioprocesos han tenido un gran desarrollo en los últimos años. El grado de aproximación depende de factores como el conocimiento del comportamiento de la reacción, los datos requeridos para la construcción y validación del modelo, requerimientos computacionales y el uso que se le dará al modelo. Debido a estos y otros factores, la incertidumbre en el modelo está presente, dado que el conocimiento exacto de los parámetros del mismo no llega a ser preciso, teniendo además la posibilidad de incertidumbre en la medida, producida por ruido en la medición (Hulhoven et al., 2006). Los bioprocesos se caracterizan por un comportamiento dinámico complejo de naturaleza no lineal, con incertidumbre del modelo, variaciones de parámetros imprevisibles, etc. Además, la mayoría de las variables representativas son típicamente no accesibles para la medición on-line. En muchos procesos es importante lograr una buena estimación de la concentración de microorganismos en el biorreactor, puesto que su medición es muy costosa y probablemente no pueda ser realizada en línea, normalmente esta variable se estima a partir de la medición de la concentración de los sustratos. En este contexto, el desarrollo de algoritmos robustos y fiables para estimar variables y parámetros importantes es de interés primordial, tanto para el control de procesos como para el monitoreo (De Battista et al., 2011; Bogaerts y Vande Wouver, 2003; Wilson et al., 1998; Bastin y Dochain, 1990).

Para la estimación de estados de los sistemas dinámicos no-lineales se ha propuesto una amplia variedad de enfoques en la literatura. Los estimadores de estado no lineales de uso común en los procesos bioquímicos se pueden clasificar en exponenciales y asintóticos. La primera clase de estimadores asume un perfecto conocimiento de la estructura del modelo que incluye las cinéticas (observador de Luenberger, filtro de Kalman, observador de modos deslizantes, etc.), además tienen una tasa ajustable de convergencia hacia el verdadero estado, que se define por uno o varios parámetros de ajuste. El principal inconveniente de los observadores exponenciales es que su eficiencia depende fuertemente de la calidad del modelo (Hulhoven et al., 2006; Dochain, 2003). Por otro lado, los estimadores asintóticos simplemente confían en las leyes de conservación sin requerir el conocimiento de la cinética del proceso, que es la mayor parte del tiempo difícil de identificar con una buena precisión. Sin embargo, el mayor inconveniente que presentan es que la tasa de convergencia está completamente determinada por las condiciones experimentales, es decir, la tasa de dilución, lo cual puede conducir a una convergencia lenta cuando el biorreactor se hace funcionar con una baja tasa de dilución o no convergencia en absoluto en el caso de cultivos discontinuos. De acuerdo con el modelo cinético y la tasa de dilución, una de estas dos clases de observadores debe ser elegido. Sin embargo, conocer a priori la calidad del modelo cinético no es trivial. Esta dificultad ha llevado al diseño de observadores de estado teniendo en cuenta la posible incertidumbre del modelo (López et al., 2013; Mesbah et al., 2011; Saleh et al., 2009; Zhang, 2009; Botero y Álvarez, 2009; Hulhoven et al., 2006; Alcaraz et al., 2004; Tham et al., 2003; González et al., 2001).

La comparación del desempeño de estimadores de estado no lineales para estimación de la concentración de biomasa a partir de la medición del sustrato en un bioproceso permite a los usuarios de estos modelos tener herramientas para la elección del mejor algoritmo de estimación de estado. Además, permite tener claro cuales estimadores rechazan muy bien las perturbaciones y cuáles no. Por otro lado, debido al avance en el desarrollo de los modos deslizantes y a su robustez frente a las perturbaciones y entradas desconocidas, estos han ido implementándose en los bioprocesos y su punto de comparación es el filtro de Kalman, ya que este filtro utiliza un proceso de optimización o minimización de los valores del error de los estados (López et al., 2013; Mesbah et al., 2011; Botero y Álvarez, 2009).

Este trabajo estudia la estimación de estados por estimadores exponenciales. Se exponen brevemente las características del bioproceso, los estimadores de estado implementados (Luenberger extendido, filtro de Kalman extendido y modos deslizantes de primer orden) y los índices de error utilizados para evaluar los estimadores, además se muestran los resultados de la simulación de los estimadores implementados y sus índices de error obtenidos. El observador de modos deslizantes implementado es de primer orden debido a: facilidad de implementación, alta capacidad de rechazar perturbaciones desconocidas, filtración de señales, baja exigencia computacional y rápida convergencia.

\section{MÉTODO}

El biorreactor continuo de tanque agitado se muestra en la Fig. 1. El modelo del sistema fue tomado de Trejos et al. (2009) y considera dos variables de estado: concentración de microorganismo y concentración de sustrato. El modelo asume mezcla perfecta dentro del tanque, el nivel de líquido en el tanque está perfectamente controlado, se asumen valores constantes de los parámetros y la cinética de crecimiento microbiano tiene inhibición por sustrato. 


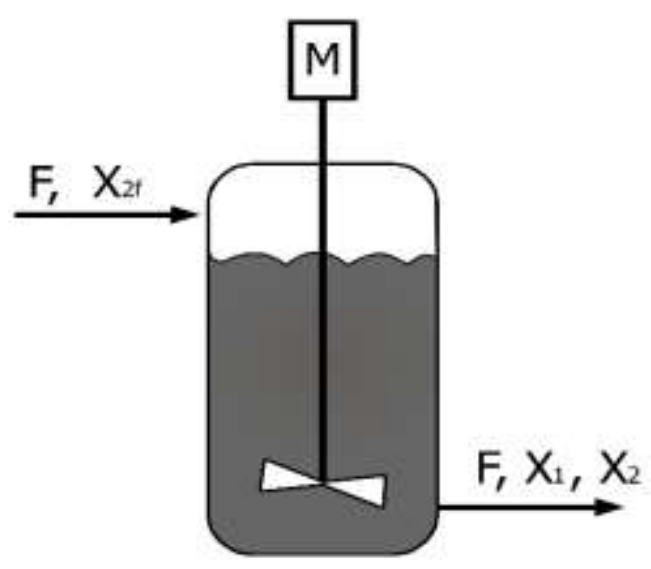

Fig. 1: Diagrama del proceso del biorreactor de tanque agitado.

Las ecuaciones del biorreactor son:

$$
\begin{aligned}
& \frac{d x_{1}}{d t}=(\mu-D) x_{1} \\
& \frac{d x_{2}}{d t}=D\left(x_{2 f}-x_{2}\right)-\frac{\mu x_{1}}{Y_{x / s}}
\end{aligned}
$$

La velocidad específica de crecimiento está expresada por el modelo de Haldane descrito en Trejos et al. (2009):

$$
\mu=\mu_{0} \frac{x_{2}}{\left(K_{m}+x_{2}+K_{i} x_{2}^{2}\right)}
$$

\section{Condiciones iniciales de las variables de proceso}

Concentración de Sustrato en la corriente de entrada al proceso: $4 \mathrm{~g} / \mathrm{L}$, tasa de Dilución (D): 0.3 1/h, concentración inicial de Biomasa en el reactor: $1.5302 \mathrm{~g} / \mathrm{L}$, concentración Inicial de Sustrato en el reactor: $0.1746 \mathrm{~g} / \mathrm{L}$.

\section{Parámetros de crecimiento del microorganismo}

Los valores de los parámetros son correspondientes a células de levadura en un sistema fermentativo en continuo reportados en Trejos et al., (2009): Velocidad específica máxima de crecimiento $\left(\mu_{0}\right): 0.531 / \mathrm{h}$, constante de afinidad $\left(\mathrm{K}_{\mathrm{m}}\right): 0.12 \mathrm{~g} / \mathrm{L}$, constante de inhibición $\left(\mathrm{K}_{\mathrm{i}}\right): 0.4545 \mathrm{~L} / \mathrm{g}$, rendimiento de sustrato en células $\left(Y_{x / s}\right): 0.4$.

\section{Perturbación realizada al proceso en la simulación de los estimadores}

Debido a que se está estudiando un proceso continuo, el microorganismo podrá renovarse periódicamente en el tanque por su suministro constante, de tal forma que los parámetros del microorganismo podrán permanecer constantes durante el proceso (esto es una suposición de acuerdo con la realidad), mientras que es mucho más frecuente los cambios en la concentración de sustrato en la entrada debido a la diversidad de la materia prima que se adiciona al tanque proveniente de procesos de hidrólisis enzimática de fuentes de almidón o celulosa. Por tanto, la concentración del sustrato en la alimentación se perturbó escalonadamente así: a las 7 horas disminuyó a $2 \mathrm{~g} / \mathrm{L}$, a las 14 horas aumentó a $4 \mathrm{~g} / \mathrm{L}$, a las 21 horas disminuyó a $2 \mathrm{~g} / \mathrm{L}$ y por último a las 28 horas aumentó a $4 \mathrm{~g} / \mathrm{L}$.

\section{Observabilidad del biorreactor}

En este estudio, la observabilidad no lineal fue basada sobre la observabilidad geométrica diferencial y se comprobó utilizando las ecuaciones 1,2 y 3.

Las variables involucradas en el modelo son: $\mathrm{x}_{2}$ (variable medida) y $\mathrm{x}_{1}$ (variable estimada). 


\section{Estimadores de estado}

En la simulación del biorreactor y los estimadores fue utilizado el método de integración de Euler (Simon, 2006) con paso de $0.01 \mathrm{~h}$, además se adicionó un ruido blanco a la medida con una varianza de $0.0025(\mathrm{~g} / \mathrm{L})^{2}$ y los valores iniciales de los estimadores fueron $(1,1)$. Para la inicialización del filtro de Kalman se partió con una matriz de covarianza inicial $P_{0}=\left[\begin{array}{cc}0.001 & 0 \\ 0 & 0.001\end{array}\right]$ y con varianza del ruido del modelo $Q_{c}=0.001$.

\section{Observador Luenberger Extendido (OLE).}

Para el diseño del observador del biorreactor continuo de tanque agitado se consideró el modelo de sistema de la forma:

$$
\begin{aligned}
& \dot{x}=f(x, u) \\
& y=x_{2}
\end{aligned}
$$

Donde $x=\left[x_{1} x_{2}\right]^{\top} \in R^{2}, y \in R$ y $u \in R$, $u$ es la entrada externa (concentración de sustrato en la alimentación), $x_{i}$ son funciones suaves $\mathrm{y}$ la salida medida es $\mathrm{y}=\mathrm{x}_{2}$.

EI OLE puede ser representado como:

$$
\begin{aligned}
& \dot{\hat{x}}=f(\hat{x}, u)+K(\hat{x})\left(y-\hat{x}_{2}\right) \\
& \hat{y}=\hat{x}_{2}
\end{aligned}
$$

Donde:

$\hat{\mathrm{x}}$ es el vector de variables estimadas, $\left[\hat{\mathrm{x}}_{1} \hat{\mathrm{x}}_{2}\right]^{\top}$, y $\mathrm{K}(\hat{\mathrm{x}})$ es la matriz de ganancias del observador. Realizando una buena selección de $\mathrm{K}(\widehat{x})$ se asegura una convergencia del error (Zhang, 2009).

\section{Observador de Modos Deslizantes (OMD).}

Se consideró el modelo del sistema de la forma representada en las ecuaciones 4 y 5 .

EI OMD se representó como:

$$
\begin{aligned}
& \dot{\hat{x}}=f(\hat{x}, u)+K(t) \operatorname{sign}\left(\mathrm{x}_{2}-\hat{\mathrm{x}}_{2}\right) \\
& \hat{y}=\hat{\mathrm{x}}_{2}
\end{aligned}
$$

Donde $\mathrm{K}(\mathrm{t})$ es la matriz de ganancias del observador y se obtuvo de acuerdo al procedimiento descrito por Wang et al. (1997).

\section{Filtro de Kalman Extendido (FKE).}

Para implementar el algoritmo del FKE como estimador de los estados del proceso de crecimiento microbiano en continuo descrito anteriormente, el modelo expresado por las ecuaciones 1, 2 y 3 se expresó como un sistema no lineal discreto de la forma (Yim et al., 2012; Yam et al., 2011):

$$
\begin{aligned}
& X_{k+1}=f\left(k, X_{k}, u_{k}\right)+W_{k} \\
& Y_{k}=h\left(k, X_{k}\right)+V_{k}
\end{aligned}
$$

Donde: $X_{k}, Y_{k}$ son los estados y salidas del sistema, respectivamente, $u_{k}$ es la entrada, $W_{k}$ y $V_{k}$ son procesos estocásticos discretos que modelan la incertidumbre de los estados y las salidas del modelo del proceso y $f$ y $\mathrm{h}$ son funciones vectoriales.

El algoritmo del FKE se define mediante el conjunto de ecuaciones 12 a 16, (Simon, 2010; Ji y Brown, 2009):

$$
\begin{aligned}
& \widehat{X}_{k}^{-}=f\left(\widehat{X}_{k-1}^{-}, u_{k}, 0\right) \\
& P_{k}^{-}=F_{k}^{X} P_{k-1}^{-}\left(F_{k}^{X}\right)^{\top}+F_{k}^{W} Q_{k-1}\left(F_{k}^{W}\right)^{\top}
\end{aligned}
$$




$$
\begin{aligned}
& \mathrm{K}_{\mathrm{k}}=\mathrm{P}_{\mathrm{k}}^{-}\left(\mathrm{H}_{\mathrm{k}}^{\mathrm{X}}\right)^{\top}\left[\mathrm{H}_{\mathrm{k}}^{\mathrm{X}} \mathrm{P}_{\mathrm{k}}^{-}\left(\mathrm{H}_{\mathrm{k}}^{\mathrm{X}}\right)^{\top}+\mathrm{H}_{\mathrm{k}}^{\mathrm{V}} \mathrm{R}_{\mathrm{k}}\left(\mathrm{H}_{\mathrm{k}}^{\mathrm{V}}\right)^{\top}\right]^{-1} \\
& \widehat{X}_{k}=\widehat{\mathrm{X}}_{\mathrm{k}}^{-}+\mathrm{K}_{\mathrm{k}}\left[\mathrm{Z}_{\mathrm{k}}-\mathrm{h}\left(\widehat{\mathrm{X}}_{\mathrm{k}}^{-}, 0\right)\right] \\
& \mathrm{P}_{\mathrm{k}}=\left(\mathrm{I}-\mathrm{K}_{\mathrm{k}} \mathrm{H}_{\mathrm{k}}^{\mathrm{X}}\right) \mathrm{P}_{\mathrm{k}}^{-}
\end{aligned}
$$

Donde:

$\widehat{X}_{k}^{-}$y $P_{k}^{-}$son el vector de estado y la matriz de covarianza a posteriori y $\widehat{X}_{k}$ y $P_{k}$ son las proyecciones de los estados y la matriz de covarianza, $Z_{k}$ son las muestras, $f$ y $h$ son las funciones no lineales vectoriales, $F^{x}$ es la matriz Jacobiana de $\mathrm{f}$ respecto a los estados, $\mathrm{F}^{\mathrm{W}}$ es la matriz Jacobiana de $\mathrm{f}$ respecto de $\mathrm{W}, \mathrm{H}^{\mathrm{X}}$ es la matriz Jacobiana de $\mathrm{h}$ respecto a los estados, $\mathrm{H}^{\mathrm{V}}$ es la matriz Jacobiana de $\mathrm{h}$ respecto de $\mathrm{V}$ y $\mathrm{K}_{\mathrm{k}}$ es la ganancia de Kalman.

Este algoritmo, al ser inicializado, va actualizando los valores de la ganancia de Kalman (matriz $\mathrm{K}_{\mathrm{k}}$ ) por optimización del error, para posteriormente actualizar los valores de los estados y de la matriz de covarianza dados por las ecuaciones (15) y (16) (Simon, 2006).

\section{Índices de error de los estimadores}

El desempeño de los estimadores fue evaluado por los indicadores de error: IAE (Integral Absolute Error), ITAE (Time-weighted Absolute Error) e ISE (Integral Squared Error) definidas en Coughanowr y LeBlanc (2009) como:

$$
\begin{aligned}
& I A E=\int_{0}^{\infty}|e(t)| d t \\
& I T A E=\int_{0}^{\infty} t|e(t)| d t \\
& \text { ISE }=\int_{0}^{\infty} e(t)^{2} d t
\end{aligned}
$$

Donde e(t) es el error, definido como la diferencia entre el valor del estimador y el valor de la medida real.

\section{RESULTADOS}

\section{Observabilidad del biorreactor de tanque agitado}

La distribución de observabilidad $\left(\Delta \mathrm{S}_{0}\right)$ obtenida fue:

$$
\Delta S_{0}=\operatorname{gen}\left\{[0,1] ;\left[-\frac{\mu_{0} x_{2}}{Y_{x / s}\left(K_{m}+x_{2}+K_{i} x_{2}^{2}\right)},-D-\frac{\mu_{0} x_{1}}{Y_{x / s}\left(K_{m}+x_{2}+K_{i} x_{2}^{2}\right)}+\frac{\mu_{0} x_{1} x_{2}\left(1+2 K_{i} x_{2}\right)}{Y_{x / s}\left(K_{m}+x_{2}+K_{i} x_{2}^{2}\right)^{2}}\right]\right\}
$$

Las condiciones para el rango total son: $\mathrm{x}_{2}>0$ y $\mathrm{x}_{2} \leq \mathrm{x}_{2 \mathrm{f}}$.

De acuerdo al resultado anterior, las condiciones requeridas para que la distribución de observabilidad sea de rango total son alcanzadas en una operación normal del biorreactor, ya que las concentraciones del sustrato son pequeñas y nunca se permite que alcance el valor de cero debido a que los microorganismos morirían. Por tanto, los estimadores de estado con dinámica arbitraria pueden ser diseñados en una amplia región del espacio de estado (Simon, 2006; Dochain, 2003).

\section{Observador Luenberger Extendido (OLE)}

Para el caso particular, la ecuación 6 quedó explícitamente expresada así:

$$
\frac{d \hat{x}_{1}}{d t}=(\hat{\mu}-D) \hat{x}_{1}+K_{1}(\hat{\mathbf{x}})\left(x_{2}-\hat{x}_{2}\right)
$$


$\frac{\mathrm{d} \hat{\mathrm{x}}_{2}}{\mathrm{dt}}=\mathrm{D}\left(\mathrm{x}_{2 \mathrm{f}}-\hat{\mathrm{x}}_{2}\right)-\frac{\widehat{\mu} \mathrm{x}_{1}}{\mathrm{Y} x / s}+\mathrm{K}_{2}(\hat{\mathbf{x}})\left(\mathrm{x}_{2}-\hat{\mathrm{x}}_{2}\right)$

Donde:

$$
\widehat{\mu}=\frac{\mu_{0} \hat{\mathrm{x}}_{2}}{\left(\mathrm{~K}_{\mathrm{m}}+\hat{\mathrm{x}}_{2}+\mathrm{K}_{\mathrm{i}} \hat{\mathrm{x}}_{2}^{2}\right)}
$$

Las ganancias del observador Luenberger extendido se calcularon según la metodología explicada por Dochain (2003) y por Zhang (2009), haciendo que la dinámica del error de estimación fuera asintóticamente estable obteniéndose:

$$
\begin{aligned}
& \mathrm{K}_{1}(\hat{\mathbf{x}})=\frac{\mathrm{ad}+\mathrm{a} \mathrm{K}_{2}(\hat{\mathrm{x}})-\mathrm{cb}-\lambda_{1} \lambda_{2}}{\mathrm{c}} \\
& \mathrm{K}_{2}(\hat{\mathbf{x}})=\lambda_{1}+\lambda_{2}-\mathrm{a}-\mathrm{d}
\end{aligned}
$$

Donde:

$$
\begin{aligned}
& \mathrm{a}=\mathrm{D}-\frac{\mu_{0} \hat{\mathrm{x}}_{2}}{\left(\mathrm{~K}_{\mathrm{m}}+\hat{\mathrm{x}}_{2}+\mathrm{K}_{\mathrm{i}} \hat{\mathrm{x}}_{2}^{2}\right)} \\
& \mathrm{b}=\frac{\mu_{0} \hat{\mathrm{x}}_{1}}{\left(\mathrm{~K}_{\mathrm{m}}+\hat{\mathrm{x}}_{2}+\mathrm{K}_{\mathrm{i}} \hat{\mathrm{x}}_{2}^{2}\right)}\left(1-\frac{\hat{\mathrm{x}}_{2}}{\mathrm{~K}_{\mathrm{m}}+\hat{\mathrm{x}}_{2}+\mathrm{K}_{\mathrm{i}} \hat{\mathrm{x}}_{2}^{2}}\right) \\
& \mathrm{c}=\frac{\mathrm{D}-\mathrm{a}}{\mathrm{Y}_{\mathrm{x} / \mathrm{s}}} \\
& \mathrm{d}=\mathrm{D}+\frac{\mu_{0} \hat{\mathrm{x}}_{1}}{\mathrm{Y}_{\mathrm{x} / \mathrm{s}}\left(\mathrm{K}_{\mathrm{m}}+\hat{\mathrm{x}}_{2}+\mathrm{K}_{\mathrm{i}} \hat{\mathrm{x}}_{2}^{2}\right)}\left(1-\frac{\hat{\mathrm{x}}_{2}\left(1+2 \mathrm{~K}_{\mathrm{i}} \hat{\mathrm{x}}_{2}\right)}{\mathrm{K}_{\mathrm{m}}+\hat{\mathrm{x}}_{2}+\mathrm{K}_{\mathrm{i}} \hat{\mathrm{x}}_{2}^{2}}\right)
\end{aligned}
$$

En este trabajo se obtuvo una convergencia rápida para valores de $\lambda_{1}=1$ y $\lambda_{2}=30$. Estos valores fueron encontrados por ensayo y error teniendo en cuenta las combinaciones que arrojaron valores menores de los índices de desempeño, el valor menor de estas combinaciones fue el valor escogido de $\lambda_{1}$ y $\lambda_{2}$.

La Fig. 2 muestra la concentración del microorganismo estimada por el observador y la Fig. 3 muestra la concentración de sustrato. El observador diseñado sigue a la planta desde las 3 horas de simulación, pero el error de estimación no converge a cero durante el tiempo de proceso, lo que indica que el observador no es capaz de rechazar las incertidumbres de la medición (Zhang, 2009).

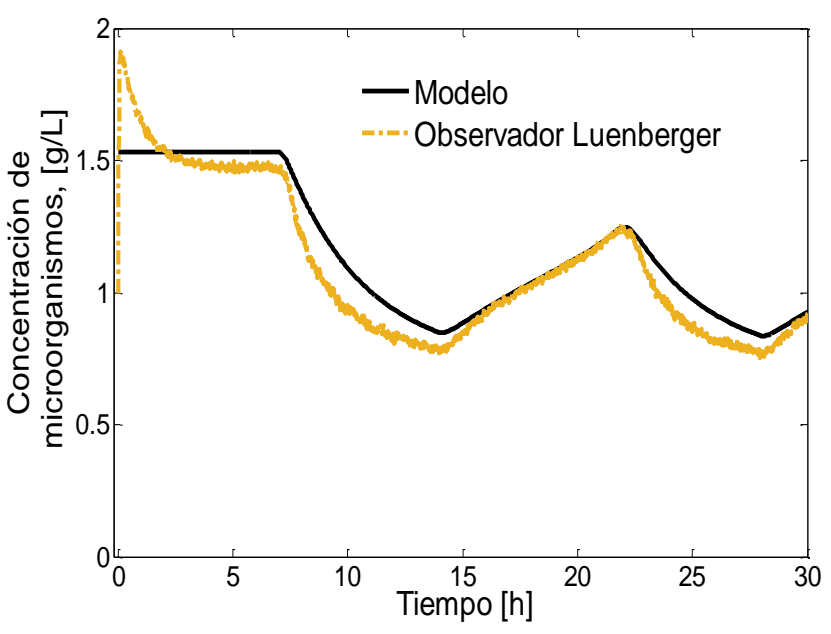

Fig. 2: Concentración de microorganismos [g/L].

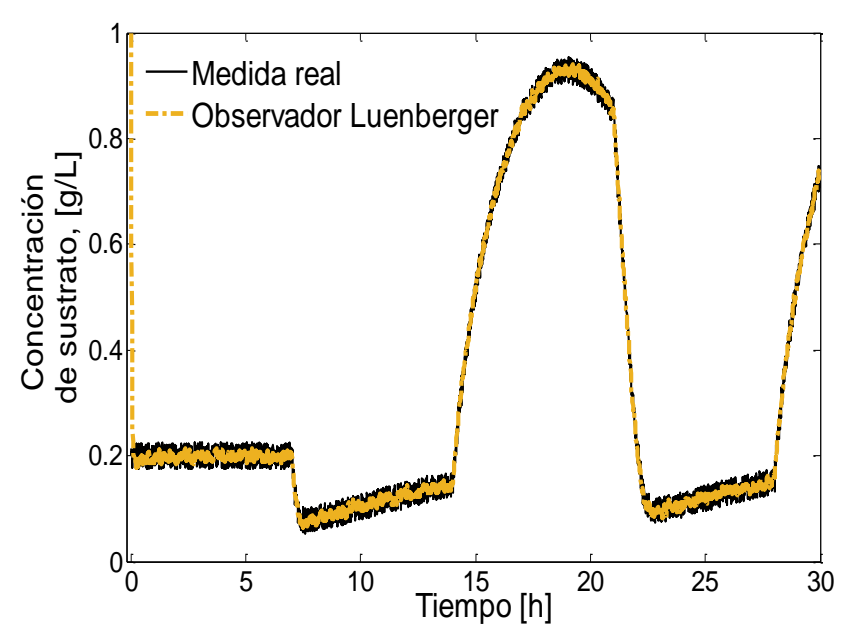

Fig. 3: Concentración de sustrato [g/L]. 
Observador de Modos Deslizantes (OMD)

Para el caso particular estudiado, la ecuación 8 quedó explícitamente expresada así:

$$
\begin{aligned}
& \frac{d \hat{x}_{1}}{d t}=(\hat{\mu}-D) \hat{x}_{1}+K_{1}(t) \operatorname{sign}\left(x_{2}-\hat{x}_{2}\right) \\
& \frac{d \hat{x}_{2}}{d t}=D\left(x_{2 f}-\hat{x}_{2}\right)-\frac{\hat{\mu} \hat{x}_{1}}{Y x_{/ s}}+K_{2}(t) \operatorname{sign}\left(x_{2}-\hat{x}_{2}\right)
\end{aligned}
$$

Donde:

$$
\widehat{\mu}=\frac{\mu_{0} \hat{\mathrm{x}}_{2}}{\left(\mathrm{~K}_{\mathrm{m}}+\hat{\mathrm{x}}_{2}+\mathrm{K}_{\mathrm{i}} \hat{\mathrm{x}}_{2}^{2}\right)}
$$

Los valores del polo del observador del polinomio deseable de primer orden $(\alpha)$ y la ganancia del observador $\left(\mathrm{K}_{1}\right)$ fueron ajustados por ensayo y error para la combinación que arrojó el menor error de estimación del observador, es decir, para $\alpha=0.3$ y $\mathrm{K}_{1}=2$. La ganancia del observador $\mathrm{K}_{2}$ se obtuvo aplicando la metodología de Wang et al. (1997) y se expresa por la ecuación:

$$
K_{2}(t)=K_{1}(t) Y_{x / s} A\left(\frac{K_{m}+\hat{x}_{2}+K_{i} \hat{x}_{2}^{2}}{\mu_{0} \hat{x}_{2}}\right)
$$

Donde:

$$
A=D-\frac{\mu_{0} \hat{x}_{2}}{\left(K_{m}+\hat{x}_{2}+K_{i} \hat{x}_{2}^{2}\right)}-\alpha
$$

En la Fig. 4 se muestra la concentración del microorganismo estimada por el observador y la Fig. 5 muestra la concentración de sustrato. Como vemos el observador sigue a la planta desde las 7 horas de simulación y el error de estimación converge a cero durante el restante tiempo de proceso. Esto indica que el observador diseñado es capaz de rechazar las incertidumbres de la medición (González et al., 2001; Wang et al., 1997).

\section{Filtro de Kalman Extendido (FKE)}

La Fig. 6 muestra la concentración del microorganismo estimada por el filtro y la Fig. 7 muestra la concentración de sustrato. El estimador sigue a la planta desde las 2.5 horas de simulación y el error de estimación converge a cero durante el restante tiempo de proceso. Esto indica que el filtro diseñado es capaz de rechazar las incertidumbres de la medición (Simon, 2006).

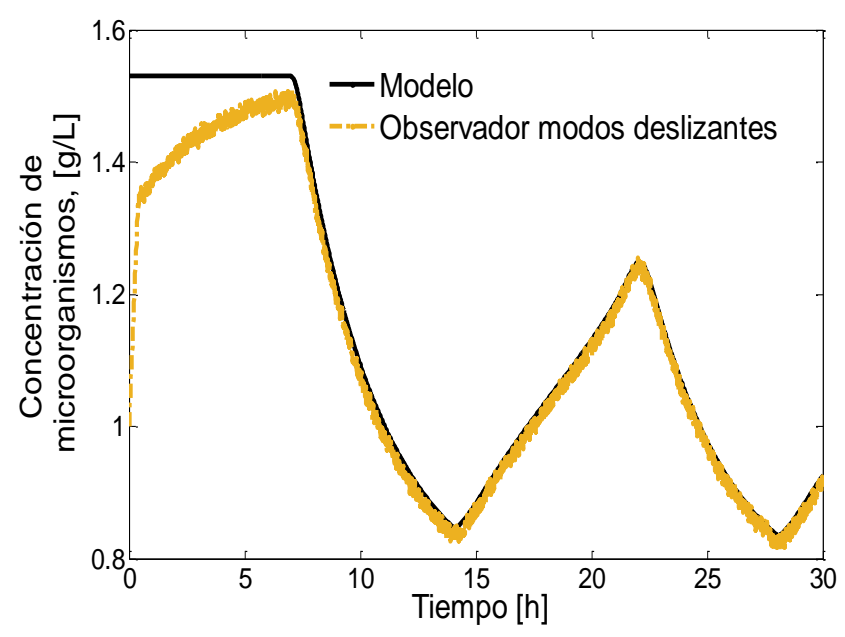

Fig. 4: Concentración de microorganismos [g/L].

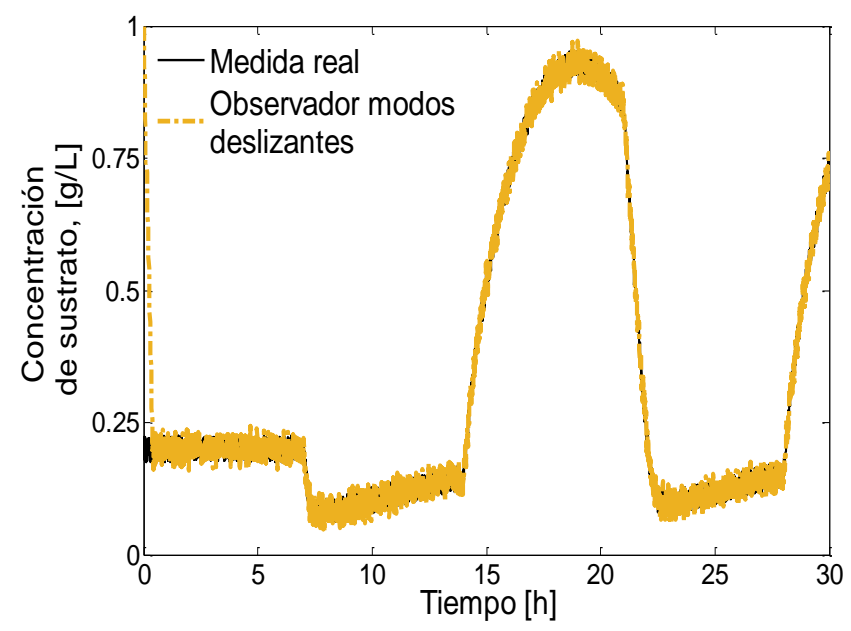

Fig. 5: Concentración de sustrato $[\mathrm{g} / \mathrm{L}]$. 


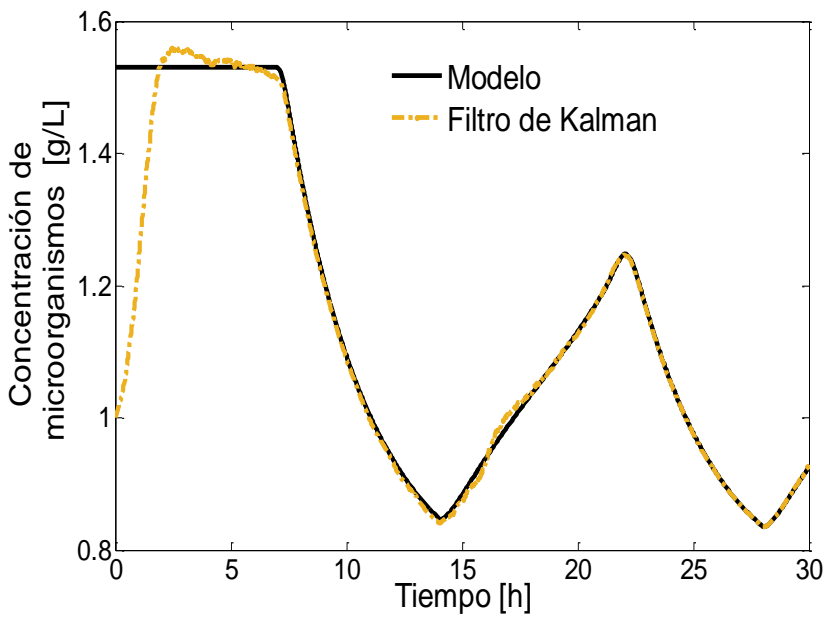

Fig. 6: Concentración de microorganismos [g/L].

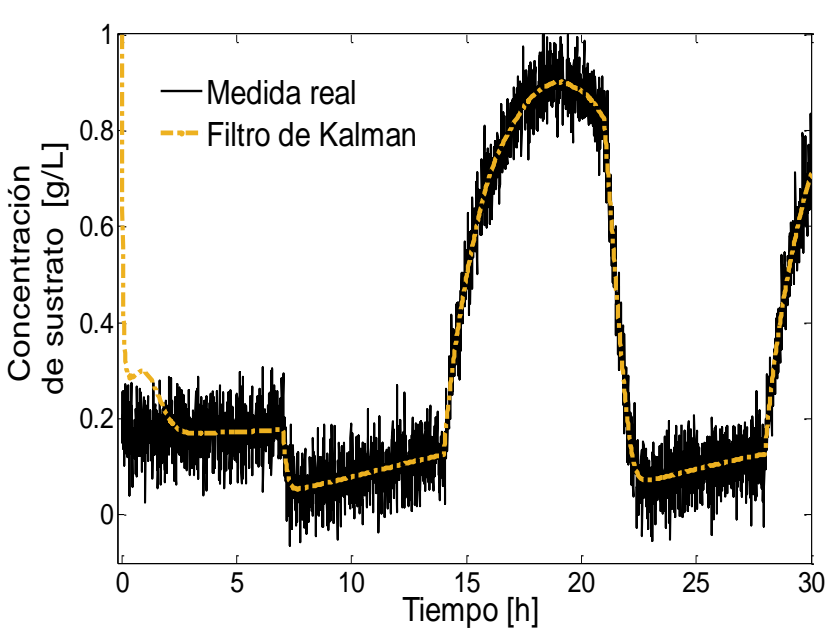

Fig. 7: Concentración de sustrato $[\mathrm{g} / \mathrm{L}]$.

Índices del error de los estimadores

La Tabla 1 muestra los índices de error de los estimadores estudiados. Se observa que el FKE fue el estimador que mejor desempeño tuvo, seguido del observador OMD. Por otro lado, el OLE no se recomienda para este tipo de procesos dado que su error de estimación es grande, como lo muestra la Fig.2 y la Tabla 1.

Aunque el estimador FKE tuvo el mejor desempeño de los tres estimadores evaluados, el usuario de los algoritmos puede utilizar el observador OMD debido a la facilidad de implementación y menor exigencia computacional que el FKE y además, los indicadores IAE e ISE del FKE y del OMD son muy parecidos.

Tabla 1: Índices del error de los estimadores

\begin{tabular}{|c|c|c|c|}
\hline Estimador & IAE & ITAE & ISE \\
\hline OLE & 2.155 & 27.757 & 0.267 \\
\hline OMD & 1.040 & 6.891 & $\mathbf{0 . 1 2 4}$ \\
\hline FKE & $\mathbf{0 . 7 0 2}$ & $\mathbf{1 . 5 5 4}$ & 0.227 \\
\hline
\end{tabular}

\section{CONCLUSIONES}

Se aplicaron tres diferentes estimadores no lineales a un sistema de crecimiento microbiano para estimar la concentración de microorganismo a partir de la medición de sustrato y como perturbación la concentración de sustrato en la entrada. El Filtro de Kalman Extendido tuvo una alta precisión de la estimación bajo condiciones de perturbación, es decir, se obtuvo una buena estimación de la biomasa $\left(x_{1}\right)$ con errores de estimación cercanos a cero. Para los demás estimadores se presentan errores de estado estacionario mayores que el FKE. El Observador de Luenberger Extendido (OLE) no se recomienda utilizar en este tipo de bioprocesos debido a los altos índices de error presentados.

\section{NOTACIÓN}

D: tasa de dilución (o el inverso del tiempo de retención) $\left(\mathrm{h}^{-1}\right)$.

$\mathrm{K}_{\mathrm{m}}$ : constante de afinidad $(\mathrm{g} / \mathrm{L})$.

$\mathrm{K}_{\mathrm{i}}$ : constante de inhibición del sustrato $(\mathrm{L} / \mathrm{g})$.

$\mathrm{x}_{1}$ : concentración del microorganismo $(\mathrm{g} / \mathrm{L})$.

x: concentración del sustrato $(\mathrm{g} / \mathrm{L})$.

$x_{2 f}$ : concentración del sustrato en la alimentación del reactor $(\mathrm{g} / \mathrm{L})$.

Yx/s: rendimiento del sustrato en microorganismo.

\section{Símbolos griegos:}

$\alpha$ : polo del observador del polinomio deseable de primer orden. 
$\lambda_{1}, \lambda_{2}$ : parámetros ajustables o sintonizables del observador de Luenberger.

$\mu$ : velocidad específica de crecimiento del microorganismo $\left(\mathrm{h}^{-1}\right)$.

$\mu_{0}$ : parámetro relacionado a la velocidad específica de crecimiento máxima del microorganismo $\left(\mathrm{h}^{-1}\right)$.

\section{REFERENCIAS}

Alcaraz, V. y otros tres autores. Observador Asintótico Ajustable para Reactores Bioquímicos: Aplicación a la Digestión Aerobia. Información Tecnológica: 15, 69-74 (2004)

Bastin, G. y Dochain, D., On-line Estimation and Adaptive Control of Bioreactors, 379. Elsevier, New York, Estados Unidos (1990)

Bogaerts, P. y Vande Wouver, A. Software sensors for bioprocesses. ISA Transactions: 42, 547-558 (2003). Botero, H. y Álvarez, H., Una revisión de los métodos más frecuentes para la estimación del estado en procesos químicos. DYNA: 72, $135-146$ (2009)

Coughanowr, D. y LeBlanc, S., Process Systems Analysis and Control, 602. McGraw-Hill, New York, Estados Unidos (2009)

De Battista, H. y otros tres autores, Specific growth rate estimation in (fed-) batch bioreactors using secondorder sliding observers. Journal of Process Control: 21, 1049-1055 (2011)

Dochain, D., State and parameter estimation in chemical and biochemical processes: a tutorial. Journal of Process Control: 13, 801-818 (2003)

Gonzalez, J. y otros cuatro autores, Sliding mode observer-based control for a class of bioreactors. Chemical Engineering Journal: 83, 25-32 (2001)

Hulhoven, X., Vande Wouver, A. y Bogaerts, P., Hybrid extended Luenberger-asymptotic observer for bioprocess state estimation. Chemical Engineering Science: 61, 7151-7160 (2006)

Ji, Z. y Brown, M., Joint State and Parameter Estimation For Biochemical Dynamic Pathways With Iterative Extended Kalman Filter: Comparison With Dual State and Parameter Estimation. The Open Automation and Control Systems Journal: 2, 69-77 (2009)

López, P. y otros cuatro autores, Software sensors design for the simultaneous saccharification and fermentation of starch to etanol. Fuel: 110, 219-226 (2013)

Mesbah, A. y otros tres autores, A comparison of nonlinear observers for output feedback model-based control of seeded batch crystallization processes. Journal of Process Control: 21, 652-666 (2011)

Saleh, S., Babaeipour, V. y Reza, A., Design of sliding mode controller for the optimal control of fed-batch cultivation of recombinant E. coli. Chemical Engineering Science: 64, 4433-4441 (2009)

Simon, D., Kalman filtering with state constraints: a survey of linear and nonlinear algorithms. IET Control Theory Appl.: 4, 1303-1318 (2010)

Simon, D., Optimal state estimation. Kalman, $\mathrm{H}^{\infty}$ y nonlinear approaches, 526. Wiley \& Sons, New Jersey, Estados Unidos (2006)

Tham, H., Ramachandran, K. y Hussain, M., Sliding Mode Control for a Continuous Bioreactor. Chem. Biochem. Eng. Q.: 17, 267-275 (2003)

Trejos, V., Fontalvo, J. y Gómez, M., Descripción matemática y análisis de estabilidad de procesos fermentativos. Revista DYNA: 158, 111-121 (2009)

Wang, G., Peg, S. y Huang, H., A sliding Observer for Nonlinear Process Control. Chemical Engineering Science: 52, 787-805 (1997)

Wilson, D., Agarwal, M. y Rippin, D., Experiences implementing the extended Kalman filter on an industrial batch reactor. Computers Chemical Engineering: 22, 1653-1672 (1998) 
Yam, H., Xia, Y. y Shi, P., A Novel Delta Operator Kalman Filter Design and Convergence Analysis. IEEE transactions on circuits and systems: 58, 2458-2468 (2011)

Yim, S., Seok, J. y Lee, J., State Estimation of the Nonlinear Suspension System based on Nonlinear Kalman Filter. 12th International Conference on Control, Automation and Systems, Jeju Island (Korea). $720-725$ (2012)

Zhang, Y., A Comparative Study of Luenberger Observer, Sliding Mode Observer and Extended Kalman Filter for Sensor less Vector Control of Induction Motor Drives. Energy Conversion Congress and Exposition. IEEE: 2466-2473 (2009) 